\title{
OPTIMUM SUPERSONIC CLIMB
}

\author{
Nguyen X. Vinh ${ }^{*}$ and Yih- Feng Tzeng ${ }^{\dagger}$ \\ University of Michigan, Ann Arbor, Michigan 48109
}

\begin{abstract}
In this paper, a simple, non-sensitive, robust, and accurate computational procedure is presented and it does not require a prior knowledge of a reference trajectory for computing the optimum trajectory for minimum-time-toclimb to a prescribed final altitude of a supersonic aircraft. From the program, we first generate the possible range of initial Mach numbers, initial altitudes and climb times for a fastest climb to a specified final altitude and Mach number while the final flight path angle is left open. Then from this family of optimum trajectories we find the relation between the minimum climb time and the specified final flight path angle for a set of given initial and final altitudes and Mach numbers, and initial flight path angle. The program routinely converges to the solution of the fastest transfer between two sets of prescribed terminal condition on altitude, Mach number and flight path angle. This program is an efficient tool for analyzing climb performance of high speed aircraft equipped with a variety of propulsion systems. In particular, climb performances between jet engine aircraft and rocket engine aircraft are compared and illustrated.
\end{abstract}

\section{Introduction}

In atmospheric flight, a minimum-time-to-climb problem leads to solving a two-point boundary value problem for a set of nonlinear ordinary differential equations. Although this problem has received much attention in the past ${ }^{1-8}$, all the known methods for its solution exhibit some deficiencies, such as less in accuracy, poor convergence, lack of robustness in the numerical computation, and most notably excessive computational cost. Moreover, a good initial reference trajectory is usually necessary for computational convergence.

In this study, we develop a non-sensitive, robust, and accurate computation scheme to solve the minimumtime-to-climb problem for a supersonic fighter aircraft from

\footnotetext{
* Professor, Department of Aerospace Engineering. Member AIAA

† Presently, Associate Scientist, Chung Shan Institute of Science and Technology, P. O. Box 90008-15-15, LungTan, Taiwan 32526, Republic of China

Copyright @ 1994 by the American Institute of Aeronautics and Astronautics, Inc. All rights reserved.
}

a set of specified initial altitude, Mach number, and flight path angle to a set of specified final altitude, Mach number, and flight path angle or free final flight path angle. From the program, we first generate the possible range of initial Mach numbers, climb times and initial altitudes for a fastest climb to a specified final altitude and Mach number. Then from this family of optimum trajectories we find the relation between the minimum climb time and the specified final flight path angle for a set of given initial and final altitudes, Mach numbers, and initial flight path angle. The program routinely converges to the solution of the fastest transfer between two sets of prescribed terminal condition on altitude, Mach number and flight path angle.

The solutions for various boundary conditions clearly display the influence of the initial Mach number and initial altitude on the optimum climb technique. In addition, the optimum control depends on the type of propulsion systems. In this respect, climb performances between jet engine aircraft and rocket engine aircraft are compared and illustrated.

\section{Equations of Motion}

Since the flight time is short for the climb, and at high speed a small angle-of-attack is used for fastest climb, except that a large negative angle-of-attack may be used in the final portion of the trajectory for a low specified final flight path angle, it is reasonable to assume in the equations that the weight is constant and that the thrust is aligned with the velocity vector. Therefore, using the standard notation, the equations of motion for climb in a vertical plane can be written as

$$
\begin{aligned}
\frac{d x}{d t} & =V \cos \gamma \\
\frac{d h}{d t} & =V \sin \gamma \\
\frac{d V}{d t} & =\frac{T-D\left(V, h, C_{D}\right)}{m}-g \sin \gamma \\
\frac{d \gamma}{d t} & =\frac{L\left(V, h, C_{L}\right)}{m ~}-\frac{g}{V} \cos \gamma
\end{aligned}
$$


The lift and the drag have the standard form

$$
\begin{aligned}
& \mathrm{L}=\frac{1}{2} \rho S \mathrm{~V}^{2} \mathrm{C}_{\mathrm{L}} \\
& \mathrm{D}=\frac{1}{2} \rho S \mathrm{~V}^{2} \mathrm{C}_{\mathrm{D}}
\end{aligned}
$$

with $C_{L}$ and $C_{D}$ being functions of the angle-of-attack and the Mach number, that is

$$
\begin{aligned}
& C_{L}=C_{L}(M, \alpha) \\
& C_{D}=C_{D}(M, \alpha)
\end{aligned}
$$

If the final range is not specified, $x$ is an ignorable variable and only the three state variables, $h, V$ and $\gamma$ are involved in the optimization process. In the dynamical system, we select the thrust $T$ and the lift coefficient $C_{L}$ as the flight controls. In the range of angle-of-attack of interest, we model the aerodynamic characteristics according to a parabolic drag polar, that is

$$
\mathrm{C}_{\mathrm{D}}=\mathrm{C}_{\mathrm{D} 0}(\mathrm{M})+\mathrm{K}(\mathrm{M}) \mathrm{C}_{\mathrm{L}}^{2}
$$

As for the thrust magnitude, it varies between a null thrust and a maximum thrust, function of the altitude and the Mach number. Then, we have

$$
0 \leq \mathrm{T} \leq \mathrm{T}_{\max }(\mathrm{h}, \mathrm{M})
$$

For the numerical example, we use the aircraft modeled in Ref. 2 for $S, C_{D 0}(M), K(M)$ and $T_{\max }(h, M)$ with $W=$ $30000 \mathrm{lbs}$. The density variation $\rho(\mathrm{h})$ for the evaluation of the aerodynamic forces and the variation of the speed of sound $\mathrm{a}(\mathrm{h})$ for the computation of the Mach number are tabulated data from the standard atmosphere but any other actual atmospheric condition can be used if enough data points are available for an accurate spline approximation in the computation of the density and speed of sound gradients.

\section{Optimum Controls}

We consider the state equations in $\mathrm{h}, \mathrm{V}$ and $\gamma$. By introducing the adjoint variables $\lambda_{h}, \lambda_{V}$ and $\lambda_{\gamma}$ we form the Hamiltonian

$$
\begin{aligned}
\mathrm{H} & =\lambda_{\mathrm{h}} \mathrm{V} \sin \gamma+\lambda_{\mathrm{V}}\left(\frac{\mathrm{T}-\mathrm{D}}{\mathrm{m}}-\mathrm{g} \sin \gamma\right) \\
& +\lambda_{\gamma}\left(\frac{\mathrm{L}}{\mathrm{mV}}-\frac{\mathrm{g} \cos \gamma}{\mathrm{V}}\right)
\end{aligned}
$$

The thrust magnitude $T$ and the lift coefficient $C_{L}$ must be selected such that at each instant the Hamiltonian is an absolute maximum. Since the thrust enters the equation for $\mathrm{V}$ linearly we have the optimum condition

$$
\lambda_{v}>0
$$

for using the maximum thrust. If $\lambda_{\mathrm{V}}<0$, the vehicle is on a coast $\operatorname{arc}$ with $T=0$. As for the optimum lift coefficient, it is given by the equation $\partial \mathrm{H} / \partial \mathrm{C}_{\mathrm{L}}=0$, which is explicitly written as

$$
\mathrm{C}_{\mathrm{L}}=\frac{\lambda_{\gamma}}{2 \mathrm{KV} \lambda_{\mathrm{V}}}
$$

The solution is then obtained by integrating the three state equations for the state vector $\vec{X}=(h, V, \gamma)$ from its prescribed initial value $\vec{X}_{0}$ and the three adjoint equations

$$
\frac{\overrightarrow{\mathrm{d} \lambda}}{\mathrm{dt}}=-\frac{\partial \mathrm{H}}{\partial \overrightarrow{\mathrm{X}}}
$$

for the adjoint vector $\vec{\lambda}=\left(\lambda_{\mathrm{h}}, \lambda_{\mathrm{V}}, \lambda_{\gamma}\right)$ while examining the sign of $\lambda_{\mathrm{v}}$ for the thrust control and using the optimum condition (8) for the aerodynamic lift control. The initial adjoint vector $\vec{\lambda}(0)$ is to be selected such that the final conditions on $h_{f}, M_{f}$ and $\gamma_{f}$ are identically satisfied.

Explicitly, we have the adjoint equations.

$$
\begin{aligned}
\frac{\mathrm{d} \lambda_{\mathrm{h}}}{\mathrm{d}}= & -\frac{\partial \mathrm{H}}{\partial \mathrm{h}}=-\frac{\mathrm{g} \lambda_{\mathrm{V}}}{\mathrm{W}}\left[\frac{\partial \mathrm{T}_{\max }}{\partial \mathrm{M}} \frac{\partial \mathrm{M}}{\partial \mathrm{h}}+\frac{\partial \mathrm{T}_{\max }}{\partial \mathrm{h}}\right. \\
& \left.-\left(\frac{\partial \mathrm{D}}{\partial \mathrm{M}}\right)_{\mathrm{V}, \mathrm{h}} \frac{\partial \mathrm{M}}{\partial \mathrm{h}}-\frac{\partial \mathrm{D}}{\partial \mathrm{h}}\right]-\frac{\mathrm{g} \lambda_{\mathrm{Y}}}{\mathrm{VW}} \frac{\partial \mathrm{L}}{\partial \mathrm{h}} \\
\frac{\mathrm{d} \lambda_{\mathrm{V}}}{\mathrm{d}}= & -\frac{\partial \mathrm{H}}{\partial \mathrm{V}}=-\lambda_{\mathrm{h}} \sin \gamma-\frac{\mathrm{g} \lambda_{\mathrm{V}}}{\mathrm{W}}\left[\frac{\partial \mathrm{T}_{\max }}{\partial \mathrm{M}} \frac{\partial \mathrm{M}}{\partial \mathrm{V}}-\frac{\partial \mathrm{D}}{\partial \mathrm{V}}\right] \\
& -\mathrm{g} \lambda_{\gamma}\left[-\frac{\mathrm{L}}{\mathrm{WV}}+\frac{1}{\mathrm{WV}} \frac{\partial \mathrm{L}}{\partial \mathrm{V}}+\frac{\cos \gamma}{\mathrm{V}^{2}}\right] \\
\frac{\mathrm{d} \lambda_{\mathrm{Y}}}{\mathrm{d}}= & -\frac{\partial \mathrm{H}}{\partial \gamma}=-\lambda_{\mathrm{h}} \mathrm{V} \cos \gamma+\lambda \mathrm{V} \cos \gamma \\
& -\frac{\lambda_{\gamma} \mathrm{g}}{\mathrm{V}} \sin \gamma
\end{aligned}
$$

Since $\mathrm{H}$ is not a function of the time explicitly, we can use the Hamiltonian integral

$$
\mathrm{H}=\mathrm{H}\left(\mathrm{t}_{\mathrm{f}}^{*}\right)=1
$$

to eliminate one of the adjoint equations. We shall use this relation to identify the unknown initial adjoint $\vec{\lambda}(0)$ as depending on only two parameters and, for generating the 
optimum trajectory, we integrate all the three equations (10) and use Eq. (11) to verify the accuracy of the solution.

By using a straight forward integration it is very difficult to obtain a physically valid solution. This is because the trajectory generated is very sensitive to change in the initial value $\vec{\lambda}(0)$ of the adjoint vector, and particularly to the value $\lambda_{r}(0)$. As shown in Fig. 1, for the case of minimum-time-to-climb between $\mathrm{h}_{0}=2 \mathrm{~km}, \mathrm{M}_{0}=$ 0.9 , and $\gamma_{0}=0^{\circ}$ and $h_{\mathrm{f}}=11 \mathrm{~km}, \mathrm{M}_{\mathrm{f}}=0.8, \gamma_{\mathrm{f}}=20^{\circ}$, a small variation in $\lambda_{r}(0)$ from the exact solution would lead to a loop or to a steep dive depending on the perturbation $\Delta \lambda_{\gamma}(0)$ to be either slightly positive or slightly negative. This can be explained by expressing $C_{L}=C_{L_{\alpha}}(M) \alpha$ and rewriting the optimum condition (8) as

$$
\alpha=\frac{\lambda_{\gamma}}{2 \mathrm{~K} \mathrm{C}_{\mathrm{L}_{\alpha}}(\mathrm{M}) \mathrm{V} \lambda_{\mathrm{V}}}
$$

Then, the perturbation in the angle-of-attack $\alpha$ is proportional to the perturbation in the adjoint variable $\lambda_{\gamma}$ and it can lead to a large deviation of the trajectory if $\lambda_{r}$ tends to deviate greatly from the exact function.

\section{Method of Solution}

For a forward integration, we need to guess two initial values for the adjoint variables while evaluating the third value from the Hamiltonian integral. Of the specified final variables $h_{f}, M_{f}$ and $\gamma_{f}$, we can use one value, say $h_{f}$, as a stopping condition for the integration and use the other two values for adjusting the unknown $\vec{\lambda}(0)$. But as mentioned above, it is very difficult to achieve a physically useful trajectory for the iteration because of the sensitivity to even a small change in the initial adjoints.

This sensitivity can be alleviated by starting with the exact values for $\lambda_{\gamma}$ and $C_{L}$. This is obtained by integrating the equations backward from a final condition on $\mathrm{h}_{\mathrm{f}}$ and $\mathrm{M}_{\mathrm{f}}$ with an arbitrary $\gamma_{\mathrm{f}}$ selected as a free parameter. Then, by the transversality condition, we have $\lambda_{x}\left(t_{f}\right)=0$ and consequently, from Eq. (8), we also have the exact value $\mathrm{C}_{\mathrm{L}}\left(\mathrm{t}_{\mathrm{f}}\right)=0$ for the lift control.

The search for the optimum trajectory connecting the initial state $\vec{X}_{0}=\left(h_{0}, M_{0}, \gamma_{0}\right)$ to the final state $\vec{X}_{\mathrm{f}}=\left(h_{\mathrm{f}}, \mathrm{M}_{\mathrm{f}}, \gamma_{\mathrm{f}}\right)$ in minimum time is achieved in two steps:

a/ By starting with $h_{f}$ and $M_{f}$, we use backward integration to solve the problem of fastest climb with free final flight path angle. Then $\gamma_{\mathrm{f}}$ is selected as a free parameter at relatively large values. The integration begins with the given $h_{f}$ and $M_{f}$, and the exact starting values $\lambda_{r}\left(t_{f}\right)$
$=0, \mathrm{C}_{\mathrm{L}}\left(\mathrm{t}_{\mathrm{f}}\right)=0$ while using $\mathrm{T}=\mathrm{T}_{\max }\left(\mathrm{h}_{\mathrm{f}}, \mathrm{M}_{\mathrm{f}}\right)$ with a second parameter $\lambda_{\mathrm{v}}\left(\mathrm{t}_{\mathrm{f}}\right)>0$ used as a scanning parameter. The remaining starting adjoint $\lambda_{h}\left(t_{f}\right)$ is evaluated from the Hamiltonian integral $\mathbf{H}=1$. The integration stops when the backward trajectory passes through the prescribed initial value $\gamma_{0}$ a second time. Since in practice, at $t=0$, we can instantaneously change $\gamma_{0}=0^{\circ}$ to any practical value for climb, we select this value as the initial value for the flight path angle. At the point where $\gamma=0^{\circ}$ for the first time, we have the initial state $\left(h_{0}, M_{0}, \gamma_{0}\right)$ to climb directly to $\left(h_{f}, M_{f}\right.$ ) while at the point where $\gamma=0^{\circ}$ for the second time, we have the initial condition such that the optimum trajectory starts with a dive to increase the speed followed by a pull up to zoom climb to the final state.

In summary, each backward integration provides two optimum trajectories, each of them leads to a set of corresponding initial altitude and Mach number, and corresponding minimum time $t_{\mathrm{f}}^{*}$. This computation procedure is simple, non-sensitive, robust, and can meet the specified boundary point precisely. For a set of specified final altitude and Mach number, by repeating this scheme, we can generate a family of optimal trajectories as shown in Figures 2 and 3 . Figure 4 is the cross plot of Figures 2 and 3. In each figure, we have the line of constant $\gamma_{f}$ with $\lambda_{\mathrm{v}}\left(\mathrm{t}_{\mathrm{f}}\right)$ as a parameter. By inspection of the plots we first notice that in the one-dive minimum-time climb there exists a critical free final flight path angle $\gamma_{c}$ such that when the free parameter $\gamma_{\mathrm{f}}>\gamma_{\mathrm{c}}$, then for a certain specified initial altitude there exist two corresponding initial Mach numbers that lead to the same specified $M_{f}, h_{f}$, and free $\gamma_{f}$ with two different minimum climb times. This has a dual result that for a certain specified initial Mach number there exist two corresponding initial altitudes that lead to the same specified $M_{f}, h_{f}$, and free $\gamma_{f}$ with two different minimum climb times. For example, for the initial Mach number $\mathrm{M}_{0}=1.003$, there exist $\mathrm{h}_{01}=2.2 \mathrm{~km}$ and $\mathrm{h}_{02}=1.1$ $\mathrm{km}$ that lead to the same final flight path angle $\gamma_{\mathrm{f}}=63^{\circ}$ with $t_{f 1}=56.885 \mathrm{sec}$ and $t_{\mathrm{f} 2}=60.18 \mathrm{sec}$ respectively as can be seen in Figures 2 and 3 . With these general data recorded, we can implement a program to routinely search for the fastest climb from any specified initial altitude $h_{0}$ and initial Mach number $\mathrm{M}_{0}$, starting with $\gamma_{0}=0^{\circ}$ and ending at the prescribed values $M_{f}$ and $h_{f}$ used to generate these two-parameter family of optimum trajectories.

In Figures 2 and 3, the point " $A$ " represents the case of minimum-time climb trajectory that just has a little dive in its beginning portion, after which it pulls up to its final conditions $\mathrm{M}_{\mathrm{f}}=0.8, \mathrm{~h}_{\mathrm{f}}=11 \mathrm{~km}$, and $\gamma_{\mathrm{f}}=60^{\circ}$. This means that this trajectory is close to a no-dive fastest climb trajectory. The point " $B$ " represents the case of minimumtime climb trajectory that has the deepest dive in its beginning portion. After diving to near sea level the trajectory begins to pull up to its final conditions. This means that the lowest altitude for this trajectory is almost zero. 
With a clear understanding of the behavior of Figures 2 and 3, we can now obtain the full range of initial altitude and Mach number for a specified final Mach number and altitude in the fastest climb problem.

Figure 5 shows the available specified region of $h_{0}$ and $\mathrm{M}_{0}$ for those fastest climb trajectories with $\gamma_{0}=0^{\circ}, h_{f}$ $=11 \mathrm{~km}$, and $\mathrm{M}_{\mathrm{f}}=0.8$. The upper curve is the boundary between the no-dive and the one-dive fastest climbs; the lower one is the natural constraint boundary which constrains those one-dive fastest climb trajectories to be above the sea level. This means that if an initial Mach number is above the upper boundary, then only those of nodive fastest climb trajectories exist. If the initial Mach number is between those two boundaries, then only the one-dive fastest climb trajectories exist.

b/ The previous analysis is the first step for solving the problem of fastest time to climb to a specified final altitude with specified final Mach number and final flight path angle. For the free final flight path angle minimum-time-to-climb problem, we have $\mathrm{C}_{\mathrm{L}}\left(\mathrm{t}_{\mathrm{f}}^{*}\right)=0$, and $\dot{\gamma}\left(\mathrm{t}_{\mathrm{f}}^{*}\right)<0$ at the final point. This is from the transversality condition and from equation (1d). Accordingly, since the free $\gamma_{f}$ is generally high, we can predict that for a specified $M_{f}, h_{f}$, and $\gamma_{f}$ problem, the point of $C_{L}\left(t_{m}\right)=0$ and $h\left(t_{m}\right)<h_{f}$ will occur at a time $t_{m}$ before the specified $\gamma_{f}$ is met. Based on this conjecture, which will be verified numerically, we develop a logical, efficient, and robust computation algorithm to meet the specified two point boundary conditions precisely. Those steps are as follows:

(1) By choosing a set of $h_{m}$ and $M_{m}$, which are generally slightly smaller and larger than $h_{f}$ and $M_{f}$ respectively, we follow the procedures described in the previous section to meet the specified initial conditions. This step will also provide $\gamma_{\mathrm{m}}$.

(2) Then, by forward integration from $h_{m}, M_{m}$, and $\gamma_{m}$, we check the resulting value of $M$ at $h=h_{f}$. If the value of $M\left(t_{f}\right)$ is not within the desired accuracy requirement, then adjust $M_{m}$ only in the forward integration to meet $\mathrm{M}_{\mathrm{f}}$, and go to step (1).

(3) A new value for $\gamma_{m}$ is obtained and proceed as in step (2) but this time check the condition on $\gamma_{\mathrm{f}}$ and adjust $h_{m}$ if necessary.

This procedure can be automatically adjusted to converge to the desired trajectory. In general, the steps (2) and (3) will be satisfied after the second or third iteration with good accuracy. In this study, the end-point accuracy is within $\mathrm{M}_{\mathrm{f}} \pm 0.005$ and $\mathrm{h}_{\mathrm{f}} \pm 0.005 \mathrm{~km}$. In Figure 6 we have shown the full range of the intermediate values for $h_{m}$ and $M_{m}$ as functions of $\gamma_{f}$ for the fastest climbs between $h_{0}$ $=2 \mathrm{~km} \mathrm{M} \mathrm{M}_{0}=0.9, \gamma_{0}=0^{\circ}$, and $h_{\mathrm{f}}=11 \mathrm{~km}, \mathrm{M}_{\mathrm{f}}=0.8$.

\section{Characteristics of Optimum Climb}

For fastest climbs, the distinctive piloting technique of starting with a dive to pick up sufficient speed for a zoom climb to the final altitude is well known. This phenomenon is mainly due to the effects of the altitude and the Mach number on the maximum thrust as shown in the constraint (5). The present computational procedure can be efficiently used for a quantitative analyses of these effects.

Figure 7 presents several optimum climb trajectories for our example aircraft, all with the same initial flight path angle $\gamma_{0}=0^{\circ}$, and the same final Mach number $\mathrm{M}_{\mathrm{f}}=0.8$. The common final altitude for the cases (a) and (b) is $h_{f}=11 \mathrm{~km}$ while the final altitude for the cases (c), (d), (e) and (g) is $h_{\mathrm{f}}=16 \mathrm{~km}$. All the final flight path angles are free, except for the last case, it is required to be $\gamma_{\mathrm{f}}=0^{\circ}$.

The starting altitude for case (a) is $h_{0}=1 \mathrm{~km}$ with an initial high Mach number $\mathbf{M}_{0}=1.1$. This results in a relatively high starting maximum thrust and the trajectory only requires a shallow dive for a few seconds before the pull-up. The climb time is $\mathrm{t}_{\mathrm{f}}=\mathbf{5 3 . 6 2}$ seconds.

The starting altitude for case (b) as for all other remaining cases is $h_{0}=3 \mathrm{~km}$. In case (b) the initial Mach number is low, at $\mathbf{M}_{0}=0.8$. This requires the aircraft to have a longer and steeper dive to reach essentially the same condition as the initial condition in case (a) before the actual pull-up. This adds nearly 14 seconds to make a total climb time of $t_{f}=67.075$ seconds.

To make more explicit the effect of the initial Mach number on the optimum climb technique, in the next three cases (c), (d) and (e), with the same starting altitude, we take respectively $\mathrm{M}_{0 \mathrm{c}}=1.1, \mathrm{M}_{0 \mathrm{~d}}=1.2$ and $\mathrm{M}_{0 \mathrm{e}}=1.3$. All the three trajectories start with a dive to the lowest altitudes at $h_{c}=2.0735 \mathrm{~km}, h_{d}=2.427 \mathrm{~km}$ and $h_{e}=2.844$ $\mathrm{km}$ respectively with the corresponding times at $\mathrm{t}_{\mathrm{c}}=24.615$ $\mathrm{s}, \mathrm{t}_{\mathrm{d}}=19.63 \mathrm{~s}$ and $\mathrm{t}_{\mathrm{e}}=11.805 \mathrm{~s}$ respectively. The resulting fastest climb times are $t_{\mathrm{fc}}=81.915 \mathrm{~s}, \mathrm{t}_{\mathrm{fd}}=74.23 \mathrm{~s}$ and $t_{\mathrm{fe}}=65.505 \mathrm{~s}$. The extra times taken in cases (c) and (d) as compared to case (e) are essentially the time to make up the insufficiency in the initial Mach number.

Finally, in case $(g)$, we use the same terminal conditions as in case (d) except that now instead of a free final flight path angle which results in $\gamma_{\mathrm{fd}}=68.2^{\circ}$, we impose the condition of prescribed final flight path angle $\gamma_{\mathrm{fg}}=0^{\circ}$. The minimum time now is $\mathrm{t}_{\mathrm{fg}}=81.87 \mathrm{~s}$. The extra time of 7.64 seconds is due to the fact that during the last portion of the climb, the rate of climb decreases to zero at the final point to meet the prescribed condition. 
Figure 8 presents the variations of the Mach number for the various trajectories generated. As seen in the figure the maximum Mach number reached is about the same for all the trajectories leading to the same final altitude and the same final Mach number.

The optimum management of the Mach number is designed to maintain a high thrust level for climb. Since for airbreathing engine, the thrust decreases as the altitude increases and since at high speed the thrust increases with the Mach number, for aircraft with jet engines it is important to have high Mach number before starting the climb. It is obvious that the higher is the final altitude, the higher is required the maximum Mach number as seen in Figure 8. But if we use a rocket engine we benefit of constant thrust during the whole trajectory. Then, in this case, the piloting technique is geared toward satisfying the final condition in the shortest time without regard to managing the thrust produced. Figure 9 shows the trajectories for fastest climb from $h_{0}=2 \mathrm{~km}, \mathrm{M}_{0}=0.9, \gamma_{0}$ $=0^{\circ}$ to $h_{\mathrm{f}}=11 \mathrm{~km}, \mathrm{M}_{\mathrm{f}}=0.8, \gamma_{\mathrm{f}}=20^{\circ}$ for the example aircraft with jet engine and with rocket engine producing the same average thrust. The comparison is to essentially show that it is not necessary for the rocket engine aircraft to engage into a dive before the pull-up. Although in a serious comparative study the analysis is much more involved due to the rating of the thrust produced, the variation of the weights during the climbs, a general rule is that rocket engine always leads to great climb rate.

\section{Conclusions}

This paper present an efficient method for calculating the minimum-time-to-climb trajectory of a supersonic aircraft between two terminal conditions on altitude, Mach number and flight path angle.

It is seen that to have high rate of climb along the trajectory, it is necessary to maintain a high level for the maximum thrust produced. Since the thrust decreases as the altitude increases during the climb and since high Mach number leads to high thrust, the optimum climb technique is to start with a dive to reach a maximum Mach number for climb. This Mach number essentially depends on the final altitude and the final Mach number.

This computational procedure is an efficient tool for analyzing climb performance of high speed aircraft equipped with a variety of propulsion systems.

\section{Reference}

[1] Rutowski, E. S., "Energy Approach to the General Aircraft Performance Problem," Journal of the Aeronautical Sciences, March 1954, pp. 187-195.

[2] Bryson, A. E., Desai, M. N., and Hoffman, W. C., " Energy-State Approximation in Performance Optimization of Supersonic Aircraft," Journal of Aircraft, Vol. 6, No. 6, Nov.-Dec. 1969, pp. 481488.

[3] Bryson, A. E., and Denham, W. F., "A SteepestAscent Method for Solving Optimum Programming Problems," Journal of Applied Mechanics, Vol. 29, June 1962, pp. 247-257.

[4] Rader, J. E., and Hull, D. G., "Computation of Optimal Aircraft Trajectories Using Parameter Optimization Methods," Journal of Aircraft, Vol. 12, No. 11, Nov. 1975, pp. 864-866.

[5] Ardema, M. D., "Linearization of the BoundaryLayer Equations of the Minimum Time-to-Climb Problem," Journal of Guidance, Control, and Dynamics, Vol. 2, No. 5, Sept.-Oct. 1979, pp. 434435.

[6] Calise, A. J., "Singular Perturbation Methods for Variational Problems in Aircraft Flight," IEEE Transactions on Automatic Control, Vol. AC-21, No. 3, June 1976, pp. 345-353.

[7] Ardema, M. D., "Solution of the Minimum Timeto-Climb Problem by Matched Asymptotic Expansions," AIAA Journal, Vol. 14, No. 7, July 1976, pp. 843-850.

[8] Enright, P. J., and Conway, B. A., "Optimal FiniteThrust Spacecraft Trajectories Using Collocation and Nonlinear Programming," Journal of Guidance, Control, and Dynamics, Vol. 14, No. 5, Sept.-Oct. 1991, pp. 981-985. 


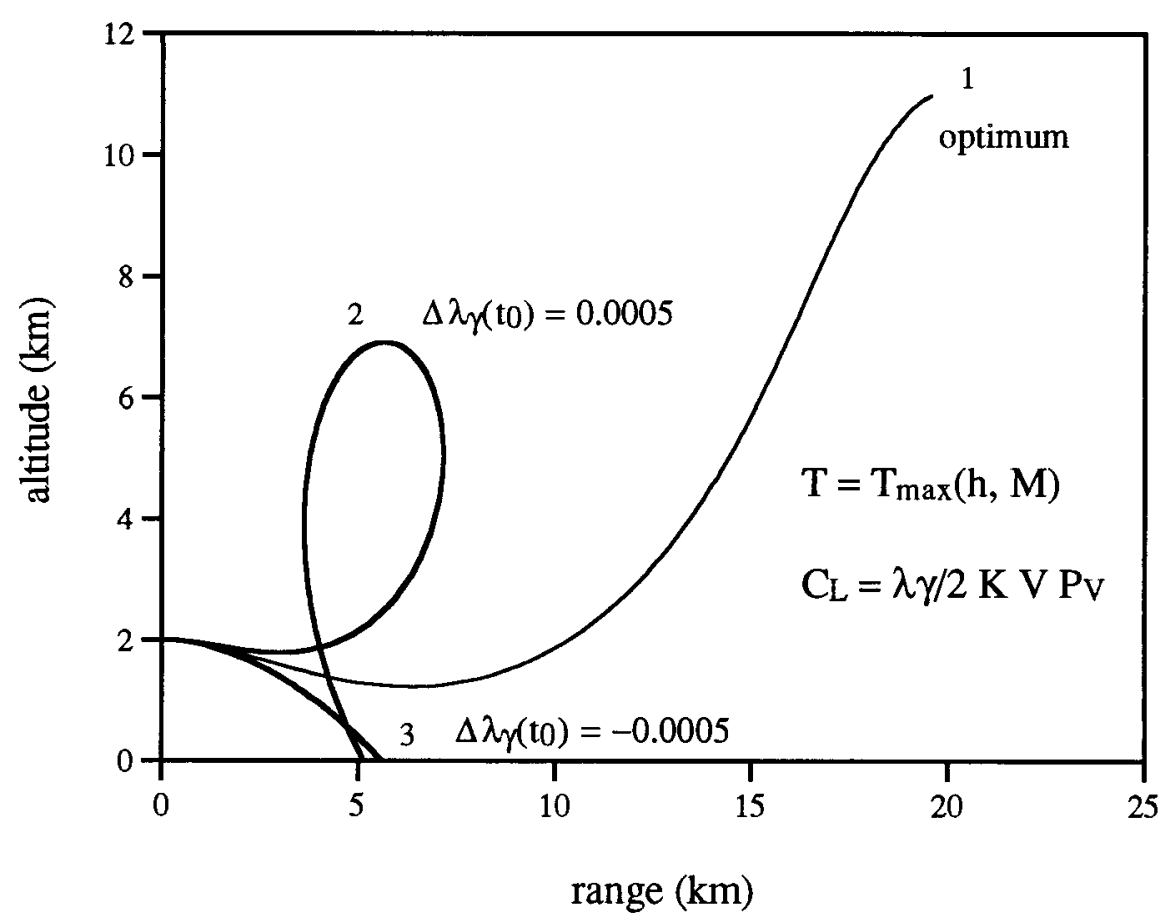

Fig. 1 The deviations of a typical zoom climb trajectory due to a small change in $\lambda_{y}(0)$

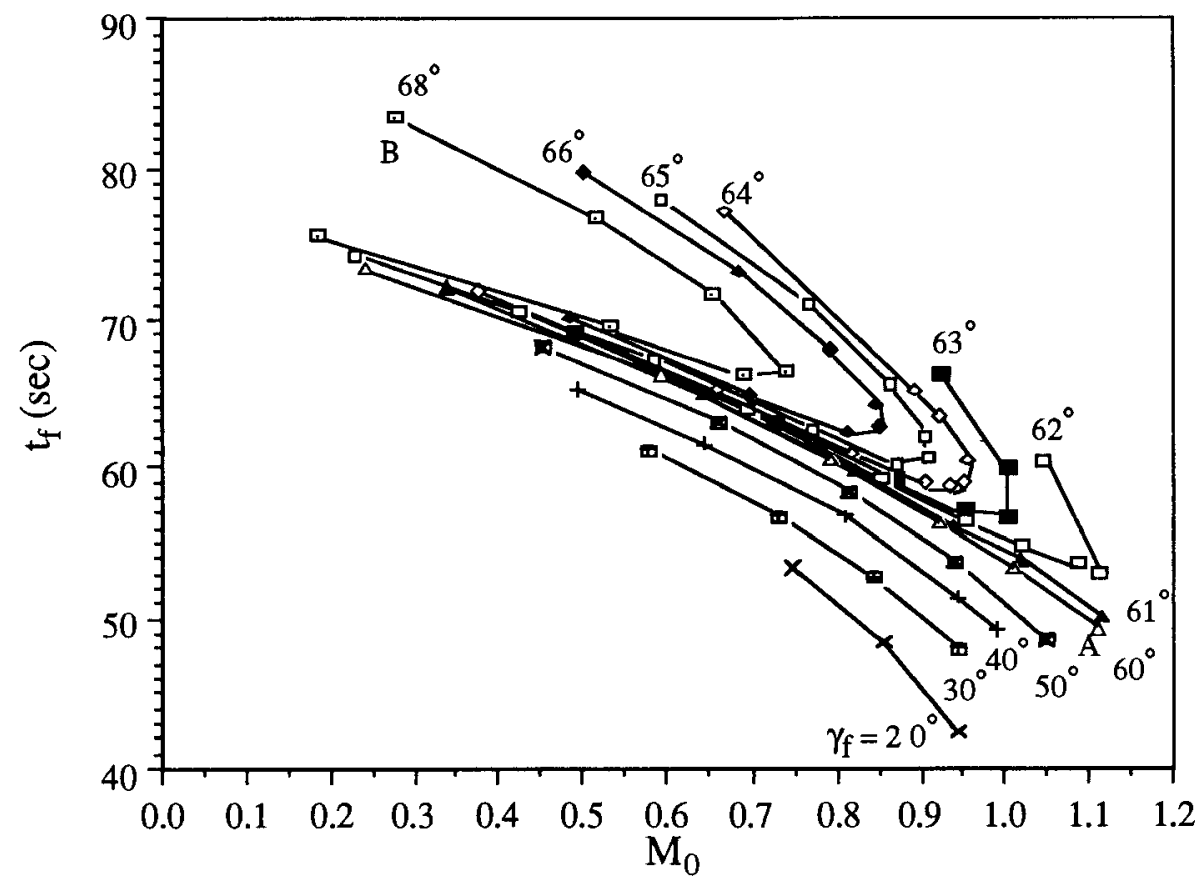

Fig. $2 \mathrm{M}_{0}$ - $_{f}$ plot for minimum-time-to-climb with one-dive (case of $h_{f}=11 \mathrm{~km}, M_{f}=0.8$ with $\gamma_{\mathrm{f}}$ as a parameter). 


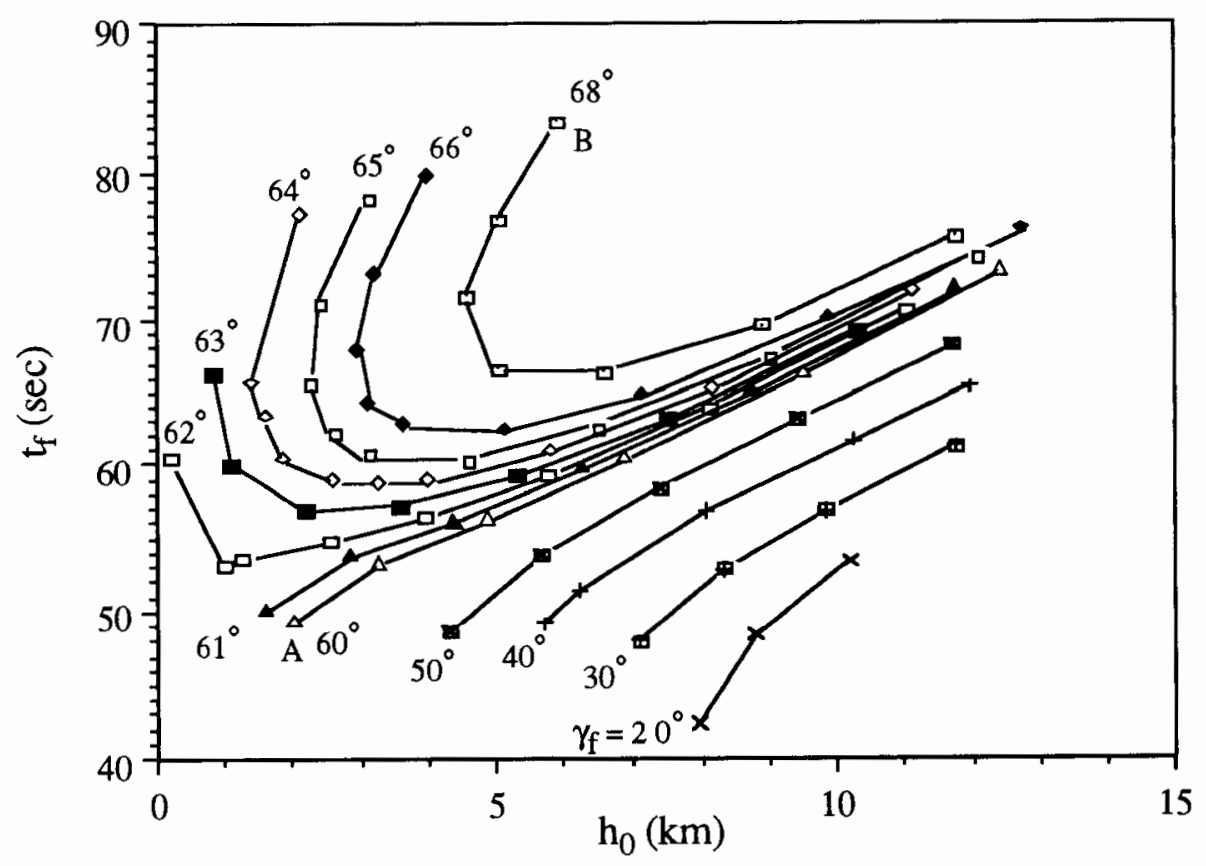

Fig. $3 \mathrm{~h}_{0}-\mathrm{t}_{\mathrm{f}}$ plot for minimum-time-to-climb with one-dive (case of $\mathrm{h}_{\mathrm{f}}=11 \mathrm{~km}, \mathrm{M}_{\mathrm{f}}=0.8$ with $\gamma_{\mathrm{f}}$ as a parameter).

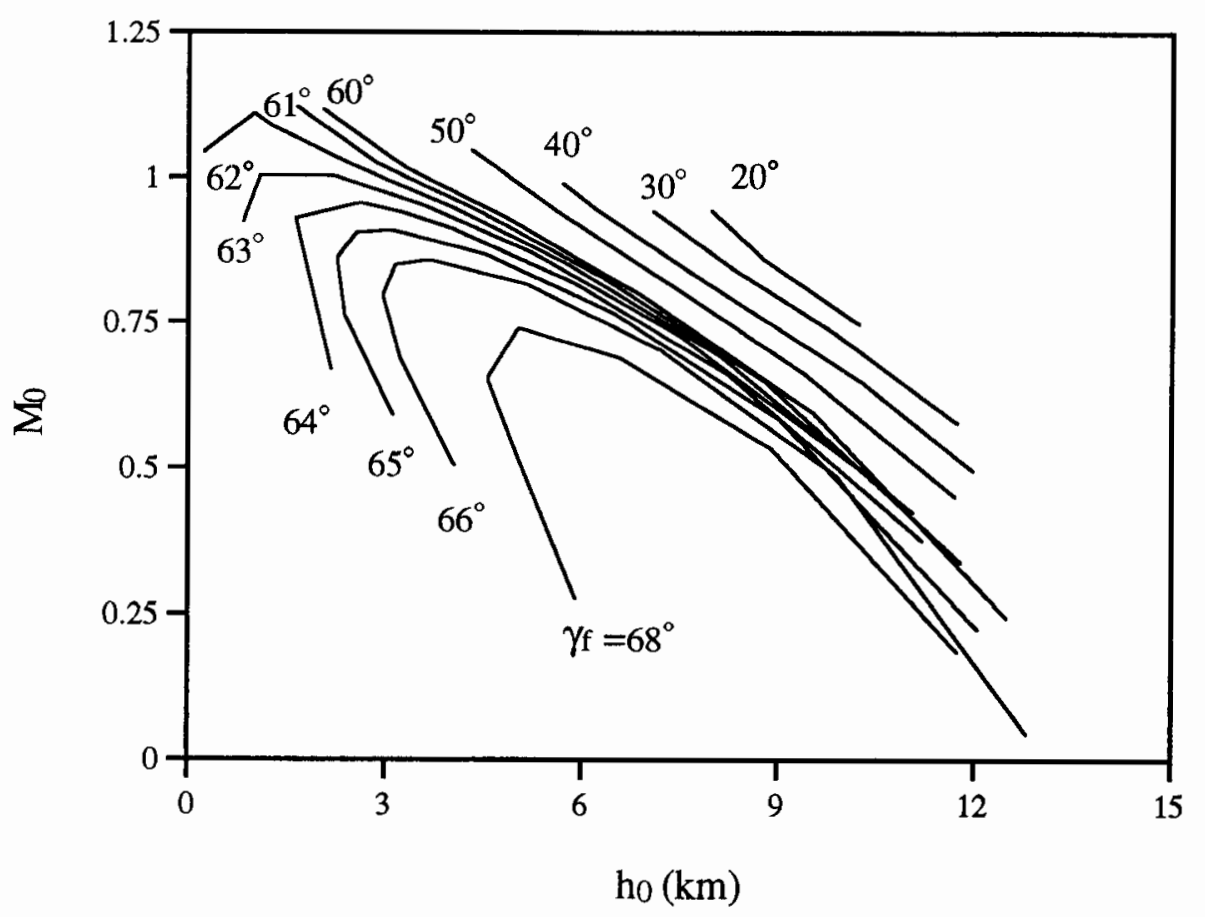

Fig. $4 \mathrm{M}_{0}$-h $\mathrm{h}_{0}$ plot for minimum-time-to-climb with one-dive (case of $h_{f}=11 \mathrm{~km}, M_{f}=0.8$ with $\gamma_{\mathrm{f}}$ as a parameter). 


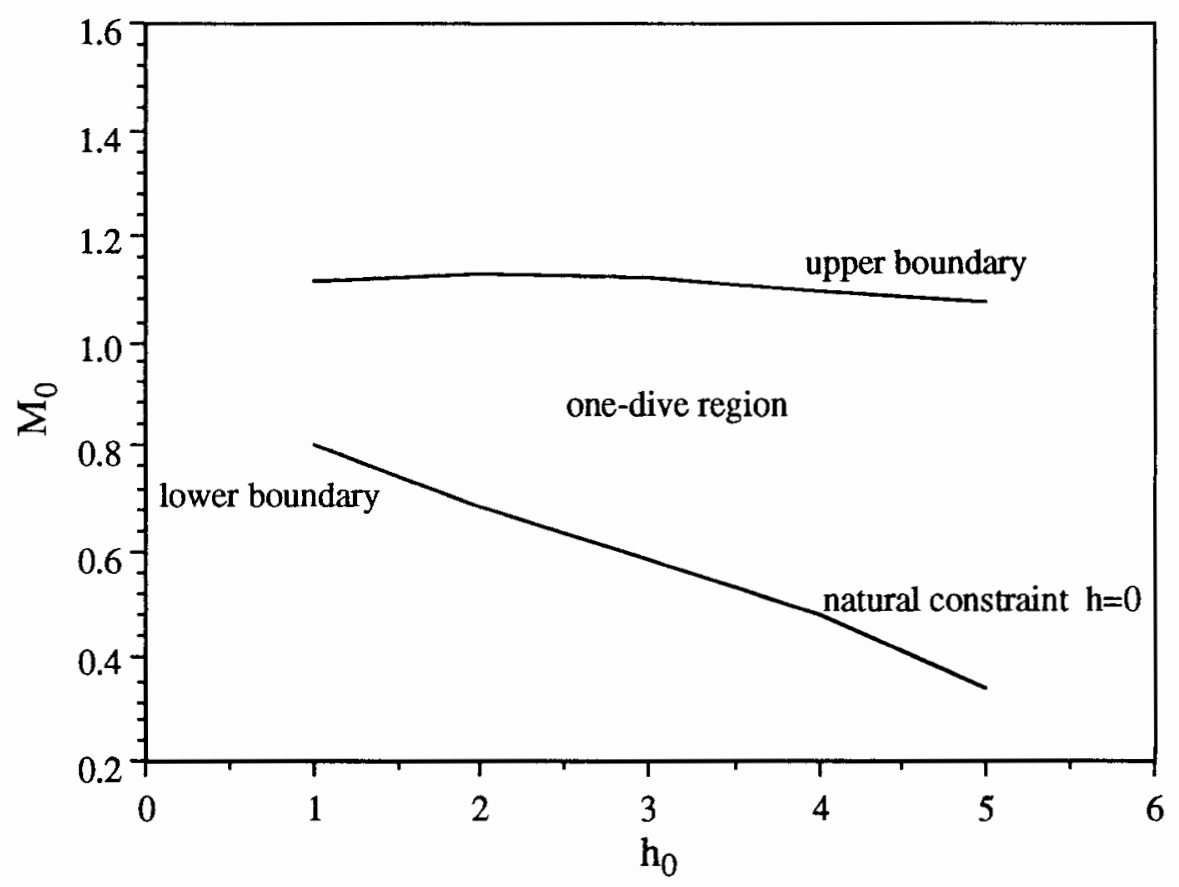

Fig. 5 Zoom climb technique as a function of the initial altitude and Mach number for fastest climb to $h_{f}=11 \mathrm{~km}, M_{f}=0.8$ and $\gamma_{f}=$ free.

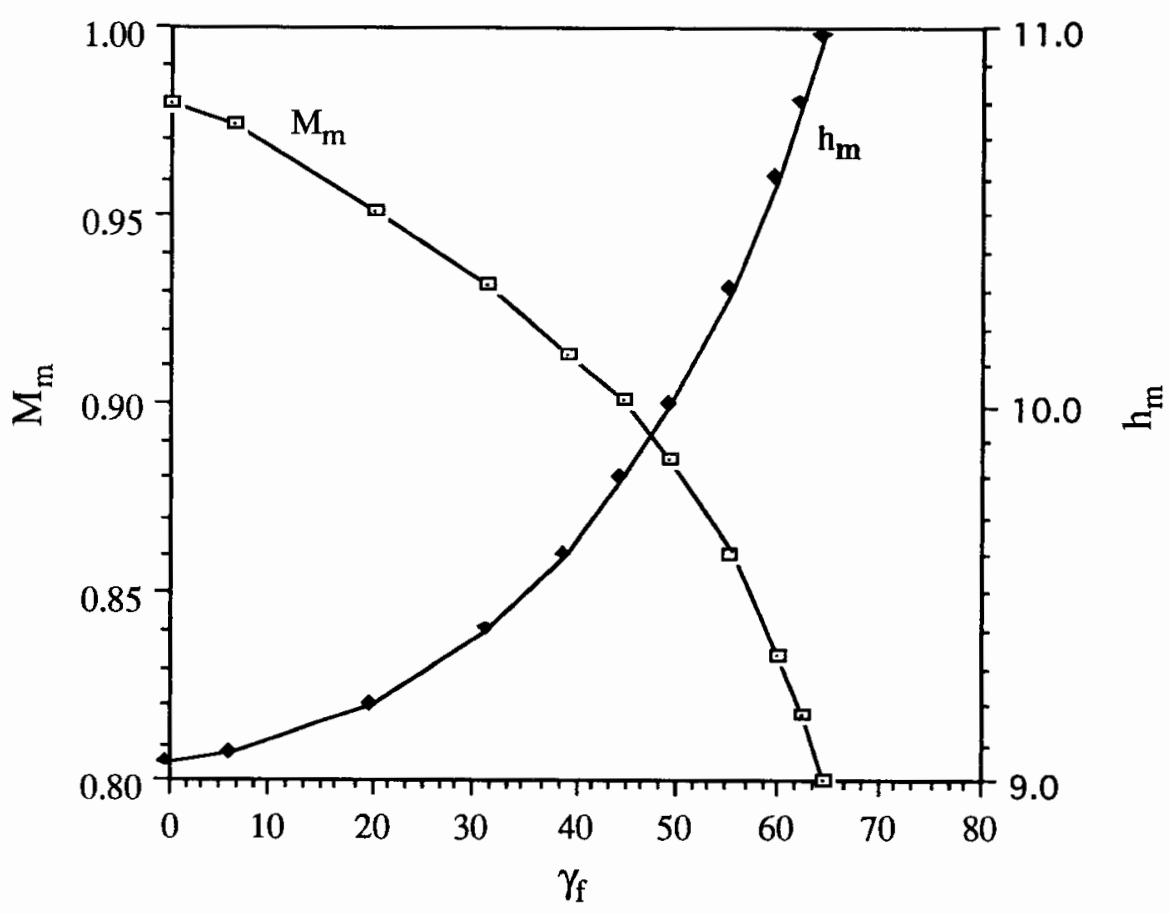

Fig. 6 Plot of $M_{m}$ and $h_{m}$ with specified $\gamma_{f}$ as a parameter 


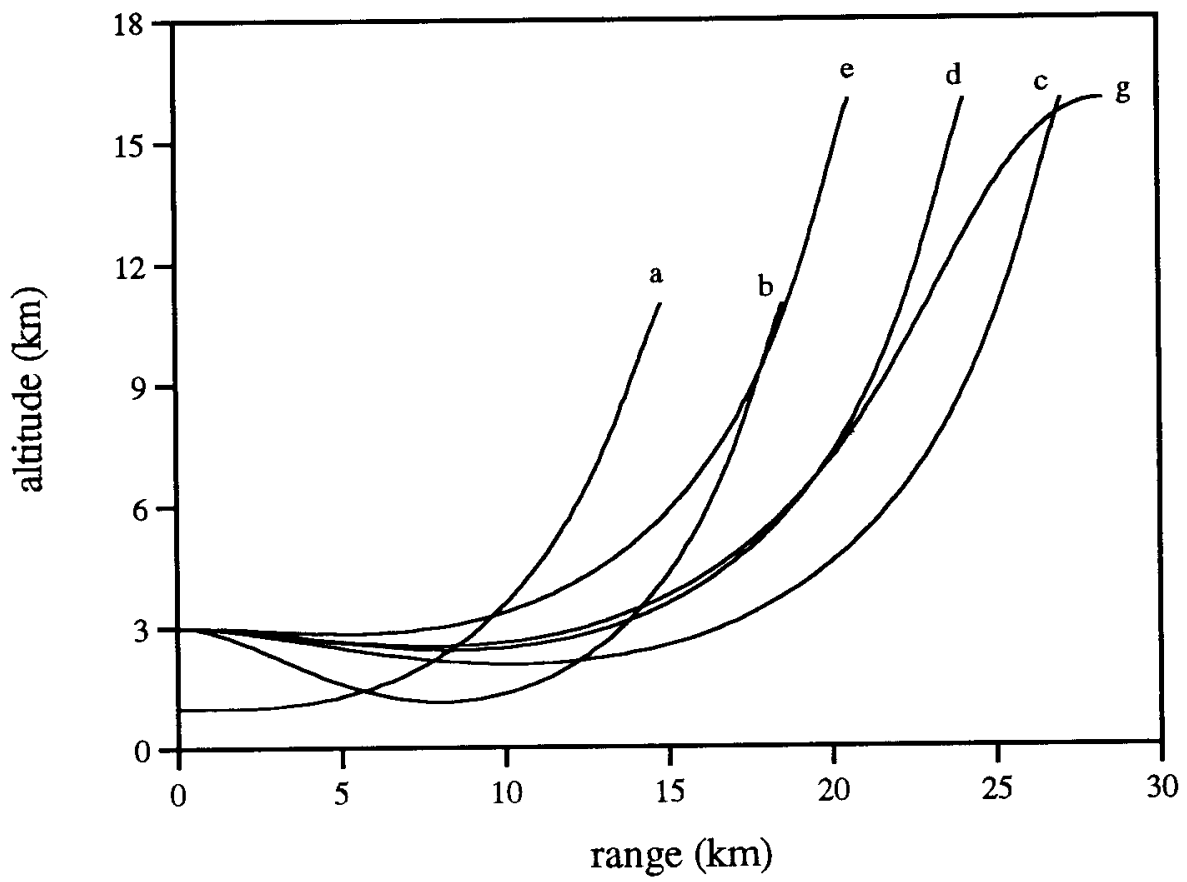

Fig. 7 Fastest climb to $h_{f}=11 \mathrm{~km}$ and $h_{f}=16 \mathrm{~km}$ with $M_{f}=0.8$

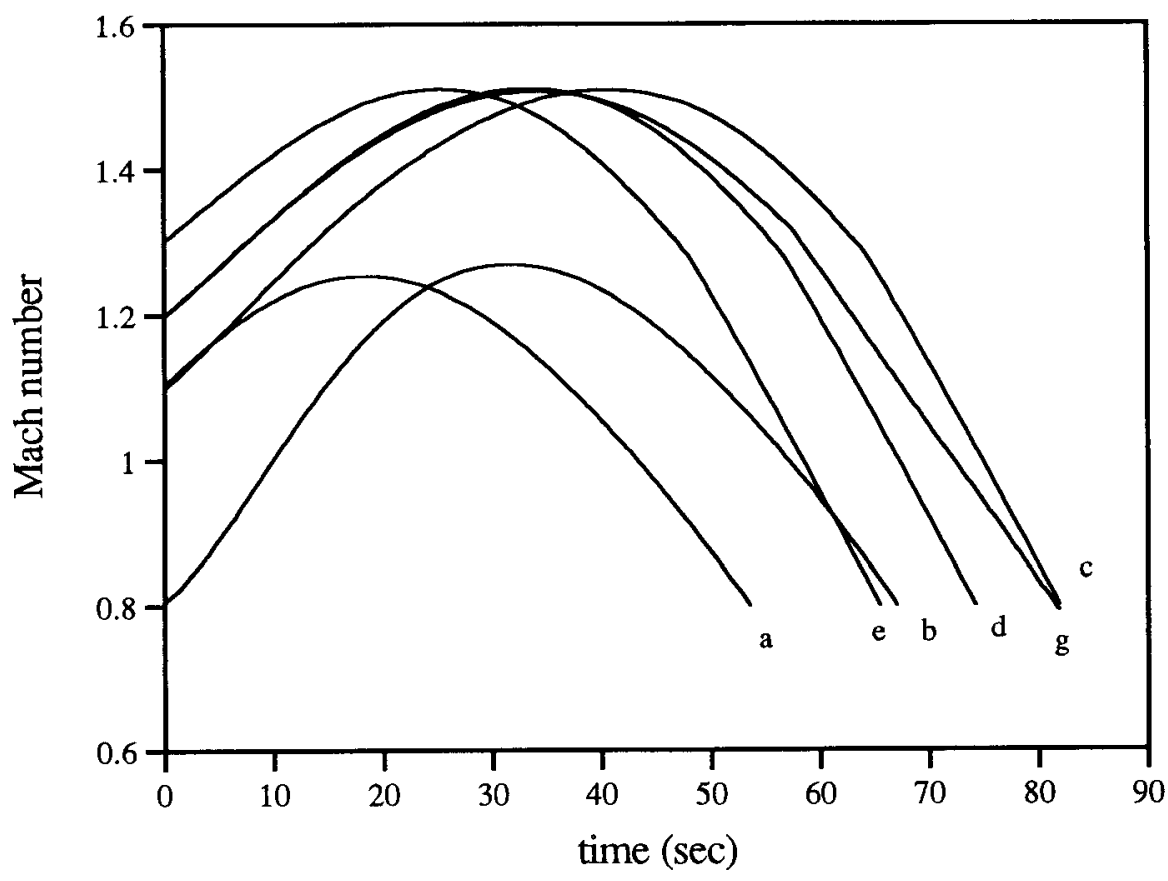

Fig. 8 Variation of the optimum Mach number. 


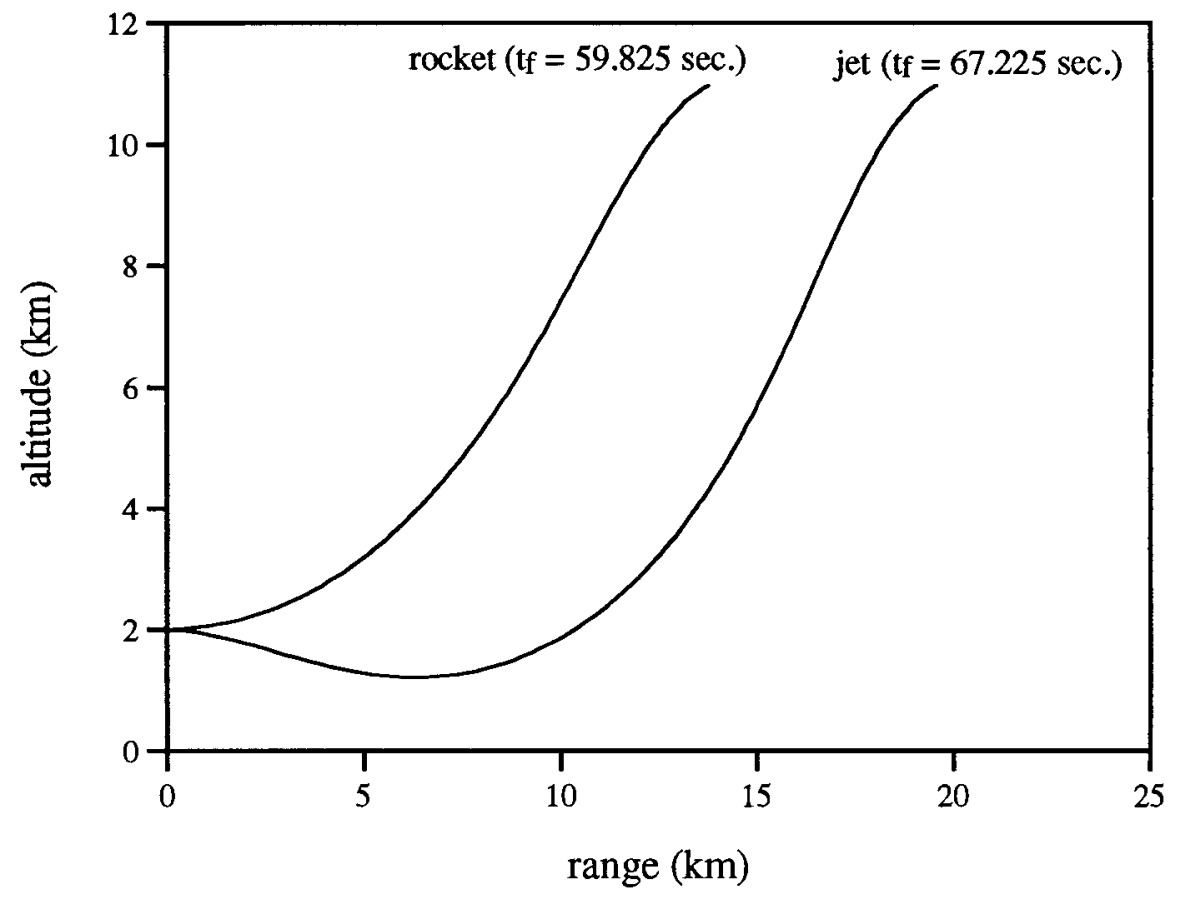

Fig. 9 Optimum trajectories with a jet engine and a rocket engine. 